\title{
Enfermagem e Psiquiatria - Enfermagem e Evolução: uma revisão de literatura
}

\author{
Nursing and Psychiatry - Trajectory and Evolution: a literature review \\ Enfermería y Psiquiatría - Trayectoria y Evolución: una revisión de la literatura
}

Geovanna Renaissa Ferreira Caldas

ORCID: https://orcid.org/0000-0001-9820-309X

Centro Universitário de Juazeiro do Norte, Brasil E-mail: geovannacaldas@hotmail.com

José Willian Lima da Silva

ORCID: https://orcid.org/0000-0003-2428-5082

Centro Universitário de Juazeiro do Norte, Brasil

E-mail: josewillianlima12@gmail.com

Vilma Regina Ferreira Rodrigues

ORCID: https://orcid.org/0000-0002-8976-5477

Hospital Regional Público da Transamazônica, Brasil

E-mail: enf.vilmarodrigues@gmail.com

Larissa Lessa dos Santos

ORCID: https://orcid.org/0000-0002-8947-6691

Universidade Estácio de Sá, Brasil

E-mail: larissalessaa@hotmail.com

Laynara dos Santos Nunes

ORCID: https://orcid.org/0000-0001-5354-5563

Universidade Federal do Ceará, Brasil

E-mail: laynara.nunes@gmail.com

Maria Zenir dos Santos Nunes

ORCID: https://orcid.org/0000-0002-1772-9266

Universidade Federal do Ceará, Brasil

E-mail: mzenir.nunes@gmail.com

Samara Atanielly Rocha

ORCID: https://orcid.org/0000-0002-5622-9280

Faculdade de Saúde e Humanidades Ibituruna, Brasil

E-mail: samaraatanielly@outlook.com

Jackson da Silva

ORCID: https://orcid.org/0000-0002-5373-9699 Centro Universitário UNIFBV, Brasil

E-mail: jacksonsilva10@bol.com.br

Ana Carolina Pinto da Silva

ORCID: http://orcid.org/0000-0002-9965-1772 Universidade Federal da Bahia, Brasil

E-mail: ana-carolina pinto@hotmail.com

Adriana Alves da Silva

ORCID: https://orcid.org/0000-0002-7361-4869

Faculdade de Ensino Superor da Amazônia Reunida, Brasil

E-mail: adrianaalvez2010@hotmail.com

Dayse Christina Rodrigues Pereira Luz

ORCID: https://orcid.org/0000-0002-5719-3574 Faculdade de Medicina do ABC, Brasi

E-mail: dayseluz.dcrp@gmail.com

Crystianne Samara Barbosa Araújo

ORCID: https://orcid.org/0000-0002-4168-7414

Centro Universitário de Juazeiro do Norte, Brasil

E-mail: crystianne.barbosa@unijuazeiro.edu.br

Cintia Nadhia Alencar Landim

ORCID: https://orcid.org/0000-0002-8189-9323

Centro Universitário de Juazeiro do Norte, Brasil

E-mail: nadhia_landim@hotmail.com

Cicero Rafael Lopes da Silva

ORCID: https://orcid.org/0000-0001-8819-5380

Centro Universitário de Juazeiro do Norte, Brasil

E-mail: rafael.lopes@unijuazeiro.edu.br

Emanuel Cardoso Monte

ORCID: https://orcid.org/0000-0002-4719-7168

Centro Universitário de Juazeiro do Norte, Brasil

E-mail: emanoelcm@gmail.com 


\author{
Alyce Brito Barros \\ ORCID: https://orcid.org/0000-0002-2775-1264 \\ Centro Universitário de Juazeiro do Norte, Brasil \\ E-mail: alyce.brito@hotmail.com \\ Maria do Socorro Santos de Oliveira \\ ORCID: https://orcid.org/0000-0001-9392-2378 \\ Centro Universitário de Juazeiro do Norte, Brasil \\ E-mail: maria.mariadosocorro.santos@hotmail.com \\ Petrúcya Frazão Lira \\ ORCID: https://orcid.org/0000-0001-9539-066X \\ Centro Universitário de Juazeiro do Norte, Brasil \\ E-mail: petrucya.frazao@unijuazeiro.edu.br
}

\begin{abstract}
Resumo
O presente trabalho teve como objetivo discorrer sobre a trajetória dos cuidados de enfermagem aos pacientes psiquiátricos, apresentando a importância da enfermagem psiquiátrica e suas mudanças antes e depois da reforma psiquiátrica. Trata-se de uma revisão integrativa da literatura realizada nas bases de dados Literatura Latino-Americana e do Caribe em Ciências da Saúde (LILACS) e na Medical Literature Analysis and Retrieval Sistem Online (MEDLINE) via Biblioteca Virtual em Saúde (BVS), com utilizaาção dos Descritores em Ciências da Saúde (DECS) em associação ao operador booleano: "Reforma psiquiátrica AND Enfermagem AND Saúde mental". Foram encontrados 98 documentos, após critérios de inclusão e exclusão, 20 estavam disponíveis, desses, 4 artigos se enquadravam na temática. A Reforma Psiquiátrica na Enfermagem acabou trazendo grandes frutos para o futuro do ensino nessa área de atuação, além de um maior acompanhamento e melhoria no ensino ao cuidado com o paciente. Evidenciou-se que o relacionamento terapêutico entre o profissional de enfermagem e o paciente terapêutico é algo mutuo e que foi desenvolvido através da evolução advinda da Reforma Psiquiátrica, permitindo um cuidado digno a esses usuários.

Palavras-chave: Enfermagem; Cuidado; Psiquiatria.
\end{abstract}

\begin{abstract}
The present study aimed to discuss the trajectory of nursing care for psychiatric patients, presenting the importance of psychiatric nursing and its changes before and after the psychiatric reform. This is an integrative literature review carried out in the Latin American and Caribbean Literature in Health Sciences databases (LILACS) and in the Medical Literature Analysis and Retrieval Sistem Online (MEDLINE) via the Virtual Health Library (VHL), with use of Health Sciences Descriptors (DECS) in association with the Boolean operator: "Psychiatric reform AND Nursing AND mental health". 98 documents were found, after inclusion and exclusion criteria, 20 were available, of these, 4 articles fit the theme. The Psychiatric Reform in Nursing ended up bringing great fruits for the future of teaching in this area of activity, in addition to greater monitoring and improvement in teaching patient care. It became evident that the therapeutic relationship between the nursing professional and the therapeutic patient is something mutual and that it was developed through the evolution arising from the Psychiatric Reform, allowing dignified care for these users.
\end{abstract}

Keywords: Nursing; Care; Psychiatry.

\title{
Resumen
}

El presente estudio tuvo como objetivo discutir la trayectoria de la atención de enfermería al paciente psiquiátrico, presentando la importancia de la enfermería psiquiátrica y sus cambios antes y después de la reforma psiquiátrica. Se trata de una revisión integradora de la literatura realizada en las bases de datos de Literatura en Ciencias de la Salud de América Latina y el Caribe (LILACS) y en el Sistema de Análisis y Recuperación de Literatura Médica en Línea (MEDLINE) a través de la Biblioteca Virtual en Salud (BVS), con uso de Descriptores de Ciencias de la Salud. (DECS) en asociación con el operador booleano: "Reforma psiquiátrica Y Enfermería Y salud mental". Se encontraron 98 documentos, luego de los criterios de inclusión y exclusión, 20 estaban disponibles, de estos, 4 artículos se ajustan a la temática. La Reforma Psiquiátrica en Enfermería acabó aportando grandes frutos para el futuro de la docencia en esta área de actividad, además de un mayor seguimiento y mejora en la enseñanza de la atención al paciente. Se hizo evidente que la relación terapéutica entre el profesional de enfermería y el paciente terapéutico es algo mutuo y que se desarrolló a través de la evolución derivada de la Reforma Psiquiátrica, permitiendo una atención digna a estos usuarios.

Palabras clave: Enfermería; Cuidado; Psiquiatría.

\section{Introdução}

Inicialmente, as práticas de saúde psiquiátrica eram aplicadas por meio do modelo biomédico, o qual possuía o profissional médico como o centro e priorizava a retraimento de pessoas com transtornos mentais, tendo como foco somente a doença, amparando a psiquiatria em manicômios, de forma que mercantilizassem tais patologias. Tais praticas frequentemente funcionavam sem a presença da enfermagem e mesmo quando presente, era exclusivamente com foco na higiene, alimentação, 
observância quanto a adesão e possíveis reações medicamentosas (Martins et al., 2018).

A concepção de doença mental como algo escrupuloso, que necessita de isolamento da sociedade, gerou preconceitos e dificuldades de inserção na comunidade. Assim, denúncias ao presente modelo emergiram, clamando por melhorias nos processos cuidativos à estas pessoas. A partir disso, surgiram os movimentos sociais com o objetivo de substituir os hospitais psiquiátricos por unidades psiquiátricas e criar um modelo que vise a humanização e atuação de equipe multidisciplinar (Sousa et al., 2016).

O movimento da Reforma psiquiátrica começou a se desenvolver a partir da década de 1980, onde as discussões sobre o modelo biomédico, passava por um franco questionamento sobre sua efetividade. A partir desse momento histórico, houve várias alterações nas políticas públicas de saúde, entre elas tínhamos a que foi considerada a maior mudança na época, que vinha a ser a criação do Sistema Único de Saúde (SUS), que ficou como modelo para o país, que trouxe junto consigo uma nova concepção para a área, tendo ao seu centro os usuários do serviço, onde foi chamada a participação dos cidadãos na gestão da assistência em saúde (Maftum et al., 2017).

A Reforma Psiquiátrica não trouxe somente mudanças nas questões organizacionais do sistema de saúde psiquiátrico, houveram também mudanças na forma de prestação da assistência a esse paciente, especialmente no atendimento de medicina e enfermagem psiquiátrica que na época eram abordados de forma mecanicista (Rodrigues et al., 2012; Mendes \& Castro, 2005).

A saúde mental, com enfoque na parte psiquiátrica, era pautada em terapias radicais, como a psicocirurgia, terapia do choque insulínico e a terapia eletroconvulsiva. Comportamentos como a repressão, punição e a negligencia na prestação do cuidado digno, faziam parte da prática assistencial, e agindo assim, aumentava-se a probabilidade de incapacitar o cliente para o retorno do convívio social (Batista, 2016).

Desse modo, salienta-se que a enfermagem psiquiátrica passou por transformações na assistência prestada ao paciente em sofrimento mental, ocorrendo a necessidade de incorporar novas técnicas voltadas à assistência psicológica e social ao tratamento do paciente (Silva et al., 2017; Esperidão et al., 2013).

Destaca-se a relevância da pesquisa por apresentar conhecimentos extremamente importante para a área de Enfermagem e de Saúde Mental, podendo futuramente proporcionar o estímulo de novas pesquisas voltadas para o assunto, pois o tema aborda um dos maiores problemas vivenciados pela humanidade a nível global, a saúde mental. Então, em relação aos profissionais de enfermagem, cabe a busca de conhecimentos sobre o assunto, para que estejam preparados sobre a temática.

Diante do exposto objetiva-se discorrer sobre a trajetória dos cuidados de enfermagem aos pacientes psiquiátricos, apresentando a importância da enfermagem psiquiátrica e suas mudanças antes e depois da reforma psiquiátrica.

\section{Metodologia}

Trata-se de uma revisão integrativa da literatura, a qual passou por um processo de seis etapas: iniciando-se pela escolha do tema e da questão que norteou o estudo; seguindo para os critérios de inclusão e exclusão dos artigos analisados; seleção das informações a serem extraídas dos estudos; a avaliação dos artigos selecionados e incluídos na revisão e por fim, a interpretação dos resultados obtidos e apresentação da revisão (Mendes et al., 2008).

Para contemplar o objetivo traçado estabeleceu-se a seguinte questão de pesquisa: Qual a mudança dos cuidados de enfermagem frente as mudanças advindas da reforma psiquiátrica?? Na perspectiva de obter dados apropriados ao propósito, realizou-se a busca nas bases de dados Literatura Latino-Americana e do Caribe em Ciências da Saúde (LILACS) e na Medical Literature Analysis and Retrieval Sistem Online (MEDLINE) via Biblioteca Virtual em Saúde (BVS), com utilização dos Descritores em Ciências da Saúde (DECS) em associação ao operador booleano na seguinte estratégia de busca: Enfermagem AND Saúde mental AND Psiquiatria, durante os meses de outubro e novembro de 2019. 
Entre os critérios de elegibilidade, foram incluídos: artigos originais, disponíveis na íntegra, com publicação nos idiomas português e/ou inglês, que abordassem a temática do estudo e possibilitassem responder à questão norteadora desta pesquisa, sendo publicados entre os anos de 2009-2019. E como critérios de exclusão: resumos, repetidos, artigos de revisões e/ou resumos.

Esta fase de seleção foi realizada de forma pareada com os pesquisadores em ambientes separados, observando inicialmente a adequação dos títulos e resumos à proposta do estudo; ao final, os pesquisadores reuniram-se para apresentar seus resultados e resolver os casos de discrepância, determinando assim os artigos a serem incluídos na revisão.

Após leitura, os dados foram inseridos em um instrumento elaborado pelos autores. Dos estudos foram extraídas informações como: autor, ano, objetivo, amostra, periódico e desfecho, das quais foram organizadas em duas ilustrações conforme a necessidade de caracterização da amostra e apresentação das principais evidências, para indicação desta última, foi utilizada a associação correspondente ao número do achado. Na etapa de avaliação, realizou-se uma análise crítica dos estudos, o que facilitou a interpretação dos dados baseando-se na literatura vigente e discussão entre os autores.

Para esclarecimento do método de busca e escolha dos documentos, utilizou-se um fluxograma baseado no protocolo PRISMA, ilustrado na Tabela 1.

Tabela 1 - Fluxograma de escolha dos estudos.
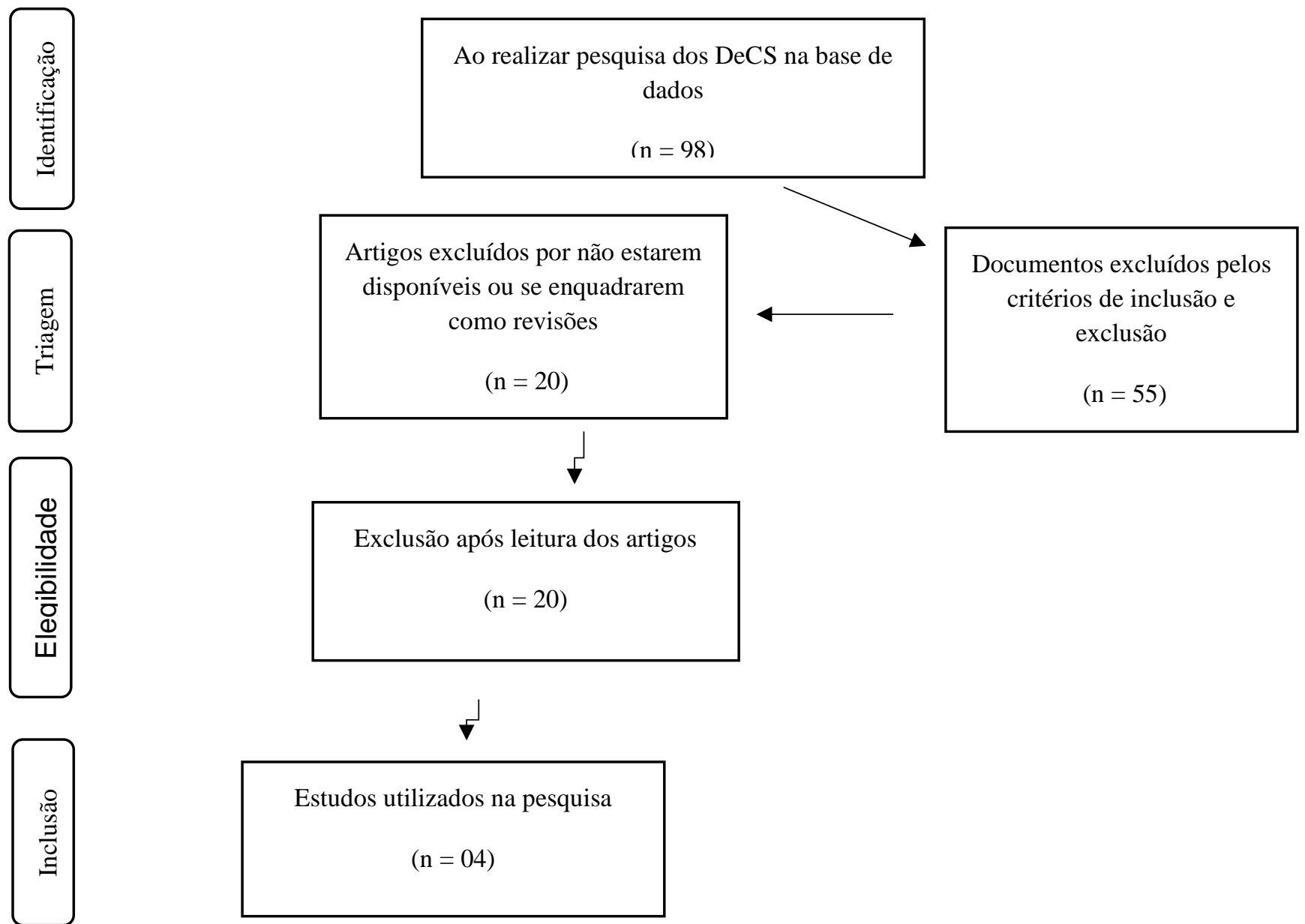

Fonte: Protocolo PRISMA 


\section{Resultados}

A partir das buscas, foram encontrados 98 artigos, após aplicação dos critérios de inclusão e exclusão definidos, restaram 55 estudos, dentre esses 20 apresentavam-se disponíveis integralmente e de forma gratuita, 08 se encontravam fora do escopo temporal, 03 não eram originais e 20 não contemplavam o objetivo do estudo por não responderem a questão norteadora ou não se enquadrarem no objetivo, restando 04 artigos incorporados a seguir:

Quadro 1 - Caracterização da amostra do estudo. Juazeiro do Norte, CE.

\begin{tabular}{|c|c|c|c|c|}
\hline $\begin{array}{c}\mathbf{N}^{0} \text { DO } \\
\text { ACHADO }\end{array}$ & AUTOR/ANO & OBJETIVO & AMOSTRA & PERIÓDICO \\
\hline 01 & $\begin{array}{l}\text { Martins GCS, } \\
\text { Peres MAA, } \\
\text { Santos TCF et } \\
\text { al. } / 2018\end{array}$ & $\begin{array}{l}\text { Analisar a atualização do ensino de } \\
\text { saúde mental em um Curso de } \\
\text { Graduação em Enfermagem, } \\
\text { concomitantemente à implantação da } \\
\text { Reforma Psiquiátrica no município de } \\
\text { Volta Redonda. }\end{array}$ & $\begin{array}{l}\text { Documentos encontrados na } \\
\text { Secretaria Municipal de Saúde } \\
\text { de Volta Redonda }\end{array}$ & $\begin{array}{l}\text { Escola Anna Nery Revista } \\
\text { de Enfermagem }\end{array}$ \\
\hline 02 & $\begin{array}{l}\text { Sousa } \quad \text { PF, } \\
\text { Maciel SC, } \\
\text { Medeiros } \\
\text { KT et } \\
\text { al. /2016 }\end{array}$ & $\begin{array}{l}\text { Conhecer e analisar as representações } \\
\text { sociais de universitários sobre a reforma } \\
\text { psiquiátrica e o doente mental, } \\
\text { relacionando-as com a adesão destes aos } \\
\text { paradigmas de atenção à saúde mental. }\end{array}$ & $\begin{array}{l}480 \text { universitários dos cursos } \\
\text { de Psicologia, Medicina e } \\
\text { Enfermagem. }\end{array}$ & $\begin{array}{lrr}\text { Publication } & & \text { of } \\
\text { Universidade de São } \\
\text { Francisco }\end{array}$ \\
\hline 03 & $\begin{array}{l}\text { Maftum MA, } \\
\text { Silva AG, Borba } \\
\text { LO et al. } / 2017\end{array}$ & $\begin{array}{l}\text { Verificar as mudanças ocorridas na } \\
\text { prática profissional decorrentes da } \\
\text { Reforma Psiquiátrica na visão da equipe } \\
\text { de enfermagem. }\end{array}$ & $\begin{array}{l}15 \text { profissionais de } \\
\text { enfermagem }\end{array}$ & $\begin{array}{llr}\text { Revista Online de } \\
\text { Pesquisa: Cuidado é } \\
\text { fundamental }\end{array}$ \\
\hline 04 & $\begin{array}{l}\text { Muniz } \mathrm{MP}, \\
\text { Tavares } \mathrm{CMM}, \\
\text { Abrahão } \\
\text { AL et } \\
\text { al. /2015 }\end{array}$ & $\begin{array}{l}\text { O objetivo foi propor uma reflexão a } \\
\text { respeito da assistência de enfermagem } \\
\text { em saúde mental e psiquiátrica. }\end{array}$ & $\begin{array}{l}\text { A metodologia utilizada foi } \\
\text { uma reflexão teórica com base } \\
\text { na Reforma Psiquiátrica } \\
\text { brasileira. }\end{array}$ & $\begin{array}{l}\text { Revista Portuguesa de } \\
\text { Enfermagem de Saúde } \\
\text { Mental }\end{array}$ \\
\hline
\end{tabular}

Fonte: Autores.

Observa-se que a reforma psiquiátrica na enfermagem acabou trazendo grandes frutos para o futuro do ensino nessa área de atuação, além de um maior acompanhamento e melhoria no ensino ao cuidado com o paciente. Alguns principais achados são descritos a seguir: 
Figura 1. Principais achados dos estudos incluídos na amostra.

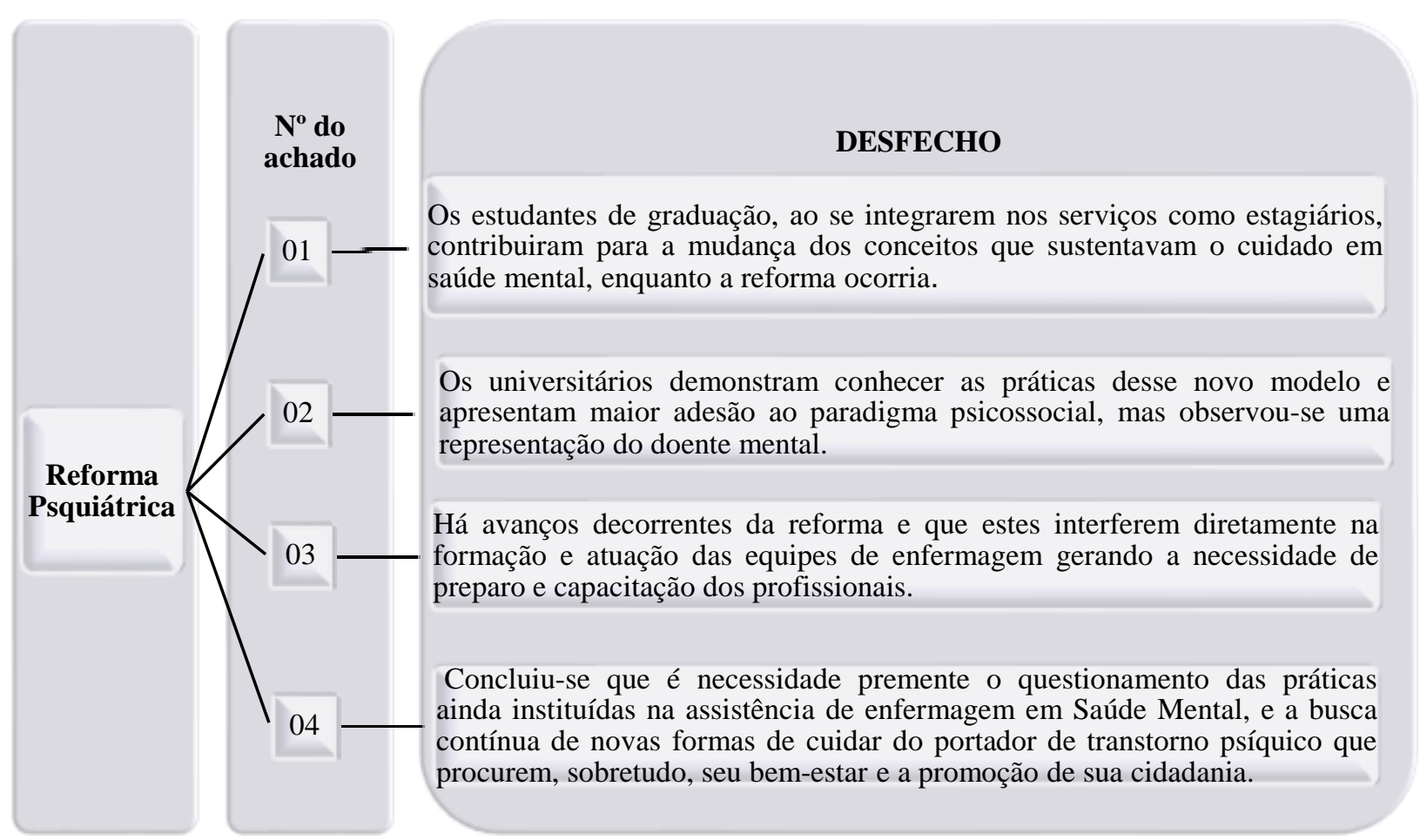

Fonte: Autores.

\section{Discussão}

Discorrendo sobre a história da psiquiatria e os preconceitos que os pacientes com transtornos sofriam, cita-se dificuldades como instituições em condições precárias, superlotações, criação de estereótipos sobre o portador do transtorno mental, salários precários e profissionais desvalorizados (Rodrigues et al., 2016).

Então, a reforma através da reorganização da assistência surgiu com o intuito de ampliar as atuações, incluindo agora reabilitação e auxílios na reinserção. Todavia, inclui-se como contrareferencia que apesar das mudanças, os profissionais devem auto perguntar se a enfermagem realmente deixou esta rotina focada em apenas cuidados básico (Muniz et al., 2015).

Um estudo realizado com a equipe de enfermagem descreve alguns relatos dos profissionais sobre as mudanças do papel da enfermagem decorrente da Reforma Psiquiátrica, salientando a evolução de ações práticas como ajudar na contenção, segurança, alimentação, higiene e de administração de medicação para uma maior participação e autonomia na tomada de decisões, permitindo a criação de grupos e o exercer de cuidados completos, de forma que a Enfermagem Psiquiátrica acompanhou e auxiliou a transformação para a visualização de um usuário ativo e com direitos. Entretanto, o profissional enfermeiro segue constantemente sobrecarregado, que por consequência colabora para um afastamento do cuidado diretamente ao usuário (Maftum et al., 2017).

Destacaram-se ainda que essas evoluções se estendem e sofrem alterações baseadas no tempo que vem sendo exercidas, nos profissionais, no estado, nos usuários e seus familiares e na sociedade no geral (Maftum et al., 2017).

Ocorre convergências sobre a atuação da equipe de enfermagem na saúde mental e da necessidade de práticas iniciativas que utilizem a criatividade na intenção de melhorar o ambiente, promover bem-estar, incluir o paciente com transtorno mental na sociedade e proporcionar um relacionamento terapêutico adequado. Dessa maneira, é necessária uma visão ampla e humanizada dos pacientes e de suas necessidades pessoais, mas também de uma bagagem de conhecimento técnico sobre as patologias, síndromes, tratamentos e modelos psicossociais, estimuladas desde os anos de graduação (Esperidão et al., 2013). 
Esse estímulo desde a graduação permite aos enfermeiros maior instigo em realizar um cuidado que inclui o acolhimento, a escuta e a interação, podendo levar em consideração o estado e as necessidades momentâneas através de uma maior relação pessoa a pessoa com uso da observação, atenção e comunicação verbal (Martins et al., 2018).

Cita-se também a ocorrência de uma contradição em sua pesquisa, onde alguns entrevistados citam o cuidado sobre os transtornos mentais como o foco de ensino e outros descrevem o cuidado do transtorno, levando em consideração a pessoa integralmente em todas as suas complexidades ocasionadas pelas patologias. Compreende-se então, que o enfermeiro passou a adentrar em algo mais dinâmico, induzido por todo o redirecionamento do cuidado a portadores de doença mental, afim de aprimorar a assistência e o formato de como os pacientes eram vistos e tratados na saúde e no quesito social (Rodrigues et al., 2016).

Foi possível ponderar a perspectiva de alguns enfermeiros sobre a importância de sair um pouco da prática e incluir a escuta durante as consultas, de forma que seja levando em consideração a carga emocional, a carga de responsabilidades, a realidade de cada paciente, acolhendo-o, dialogando, considerando a visão de cada ser, atentar para as necessidades de encaminhamentos e passar a incluir a família nas conversas, no intuito de a pessoa não ser vista apenas em um contexto, mas sim como um todo (Lima et al., 2015).

Também possibilitou o fortalecimento dessas perspectivas em outro estudo, onde descreveram a reforma como algo que veio para incluir esses pacientes em outros âmbitos, mudando o termo internação para acolhimento, deixando para trás as ações de trancar o paciente, amarrar, ter medo e evoluir para deixá-los a vontade, orientando, ajudando, sendo referência e reforçando o lado humano, tornando um antigo ambiente hospitalocentrico em um ambiente familiar humanizado (Silva \& Azevedo, 2011).

Expõe-se ainda, a incorporação de um chefe de enfermagem, onde foi acrescentado novas responsabilidades e compromissos, como promover um trabalho multidisciplinar em equipe e reestruturar o ambiente e a assistência (Moraes et al., 2010).

Complementando isso, abrange para práticas de administração, treinamento, controle, supervisão, participação em grupos terapêuticos, acolhimento, escuta, visita, cuidados básicos (banho, limpeza, alimentação, repouso), sistematização dos cuidados, grupos que estimulam o cognitivo e o contato/convívio social e avaliação geral de cada ser (Dias \& Silva, 2010).

A Reforma Psiquiátrica gerou o entendimento de que saúde mental não pode ser diminuída a patologia ou a semiologia básica, através da utilização do conceito de que a atenção ao portador de transtorno mental não se restringe a algo único, mas um complexo de abrangências. É descrito que quando uma pessoa se sente amparada de forma humana, ela contribui positivamente e ativamente na adesão ao tratamento e facilita o processo de reabilitação (Xavier et al., 2017).

O profissional além das práticas já relatadas anteriormente, pode desenvolver ações que levem em consideração os potenciais, as habilidades, mas também os medos e as limitações, cita-se como exemplo os grupos de apoio, oficinas, reuniões com as famílias, visitas domiciliares e trabalhos multidisciplinares, na tentativa de estabelecer vínculos com os pacientes (Silva et al., 2017).

Sendo assim, evidencia-se a importância de uma educação continua através de treinamentos e capacitações, de leituras de pesquisas realizadas por outros enfermeiros e a realização de estudos sobre o tema em enfoque, conferindo um bom conhecimento teórico e prático para a realização de uma assistência como algo transformador, de forma que siga os caminhos da reforma psiquiátrica (Mendes \& Castro, 2005).

\section{Considerações Finais}

$\mathrm{O}$ presente estudo trouxe a contextualização da trajetória seguida pela enfermagem dentro da psiquiatria e pelas mudanças que aconteceram por causa da reforma psiquiátrica. Buscou destacar os fatos que marcaram cada época dessa assistência sempre ressaltando a diferença da época para os dias atuais, além de mostrar como a enfermagem teve um papel 
importante dentro dos modelos psiquiátricos do Brasil, ajudando na mudança do estilo assistencial para a pessoa com sofrimento psíquico.

A figura do profissional de enfermagem na atualidade, sobretudo a psiquiátrica, cresceu em complexidade desde seus elementos históricos originais, para uma prática que ocorre em contexto social e ambiental. Evidenciando-se que o relacionamento terapêutico entre o profissional de enfermagem e o paciente terapêutico é algo mutuo e que foi desenvolvido através da evolução advinda da Reforma Psiquiátrica, permitindo um cuidado digno a esses usuários.

Espera-se que estudo posso vir a servir como base para sinalizar a importância de pesquisas dentro dessa temática apresentada, de maneira que venha a se ter mais conhecimento histórico com o propósito de não repetir os erros do passado.

Observa-se que a temática apresenta-se escassa, o que dificultou o desenvolvimento do estudo, mas que principalmente, demostra faltas de pesquisas na área, principalmente pesquisas na língua portuguesa, já que os descritores encontravam-se em português, o que facilitaria o acesso de buscas para as mesmas.

\section{Referências}

Batista, E. C. A saúde mental no Brasil e o atual cenário dos Centros de Atenção Psicossocial (CAPS). Interdiscip Rev Eletrônica UNIVAR, 16(2), 29-35.

Dias, C. B., \& Silva A. L. A. O perfil e a ação profissional da(o) enfermeira(o) no Centro de Atenção Psicossocial. Revista da escola de Enfermagem da USP, $44(2), 469-475$.

Esperidão, E., Silva, N. S., Caixeta, C. C., \& Rodrigues, J. A enfermagem psiquiátrica, a ABEn e o departamento científico de enfermagem psiquiátrica e saúde mental: avanços e desafios. Rev Bras. Enferm, 66, 171-176.

Lima, D. W. C., Vieira, A. N., \& Silveira, L. C. A escuta terapêutica no cuidado clínico de enfermagem em saúde mental. Texto contexto - Enfermagem, 24(1), 154-160.

Maftum, A. M., Silva, A. G., Borba, L. O., Brusamarello, T., \& Czarnobay, J. Mudanças ocorridas na prática profissional na área da saúde mental frente à reforma psiquiátrica brasileira na visão da equipe de enfermagem. J. Res.: Fundam Care Online, 9(2), 309-314.

Martins, G. C. S., Peres, M. A. A., Santos, T. C. F., Queirós, P. J. P., Paiva, C. F., \& Almeida, F. A. J. Ensino de graduação em enfermagem em saúde mental como aliado à consolidação do movimento de Reforma Psiquiátrica. Esc. Anna Nery, 22(4).

Mendes, K. D. S., Silveira, R. C. C. P., \& Galvão, C. M. Revisão integrativa: método de pesquisa para a incorporação de evidências na saúde e na enfermagem. Texto Contexto Enfermagem, 17(4), 758-64.

Mendes, T. H., \& Castro, R. C. B. R. Conhecimento do Enfermeiro e seu papel em psiquiatria. Rev Enferm UNISA, 6, 94-98.

Moraes, A. E. C., Almeida, F. A. J., Santos, T. C. F., Peres, M. A. A., Souza, M. C. F., \& Oliveira, A. B. Implantação da Reforma Psiquiátrica no município de Volta Redonda: Implicações para a enfermagem. Texto contexto - Enfermagem, 19(3), 526-35.

Muniz, M. P., Tavares, C. M. M., Abrahão, A. L., \& Souza, A. C. A assistência de enfermagem em tempos de reforma psiquiátrica. Revista Portuguesa de Enfermagem de Saúde Mental. 13.

Rodrigues, A. A. P., Xavier, M. L., Figueiredo, M. A. G., Almeida, F. A. J., \& Peres, M. A. A. Influências da reforma psiquiátrica no cuidado de enfermagem na casa de saúde esperança em Juiz de Fora, Minas Gerais, Brasil (1994-1998). Texto contexto - Enfermagem, 25(2),.

Rodrigues, J., Santos, S. M. A., \& Spricigo, J. S. Ensino do cuidado de Enfermagem em Saúde Mental através do discurso docente. Texto Contexto Enfermagem, 21(3), 616-624.

Silva, D. S., \& Azevedo, D. M. A Reforma Psiquiátrica na visão de quem cuida: Percepções de profissionais do serviço residencial terapêutico. Esc. Anna Nery, $15(3)$.

Silva, M. S., Machado, P. A. T., Nascimento, R. S., Oliveira, T. S., Silva, T. F., \& Batista, E. C. A Enfermagem no campo da saúde mental: Uma breve discussão teórica. Revista Amazônia Science \& Health, 5(2).

Sousa, P. F., Maciel, S. C., Medeiros, K. T., \& Vieira, G. L. S. Atitudes e Representações em Saúde Mental: Um Estudo com Universitários. Psico-USF, 21(3), $527-538$.

Xavier, M. S., Terra, M. G., Schimith, M. D., Leite, M. T., Kruse, M. H. L., \& Arnemann, C. T. Compreensão de enfermeiras atuantes em saúde mental sobre a internação compulsória e involuntária. Esc. Anna Nery, 21(3). 